\title{
Dual Antithrombotic Therapy in Atrial Fibrillation Patients undergoing Percutaneous Coronary Angioplasty: The Impact of Bleeding Risk Score on Outcome
}

\author{
Konstantinos Aznaouridis ${ }^{1} \quad$ Maria-Eirini Tselegkidi ${ }^{2}$ \\ ${ }^{1}$ 1st Department of Cardiology, Hippokration Hospital, National and \\ Kapodistrian University of Athens Medical School, Athens, Greece \\ ${ }^{2}$ Department of Clinical Therapeutics, Alexandra Hospital, National and \\ Kapodistrian University of Athens Medical School, Athens, Greece \\ 3 2nd Department of Cardiology, Attikon University Hospital, National and \\ Kapodistrian University of Athens Medical School, Athens, Greece
}

Thromb Haemost 2021;121:845-847.
Ioannis Kanakakis ${ }^{2}$ Dimitrios Alexopoulos 3

Address for correspondence Dimitrios Alexopoulos, MD, FACC, 2nd Department of Cardiology, Attikon University Hospital, Rimini 1, Chaidari 12462, Athens, Greece (e-mail: dalex@med.uoa.gr).

(PIONEER-AF PCI, REDUAL-PCI, AUGUSTUS, and ENTRUST-AF $\mathrm{PCI}) .^{5-8}$ The primary outcome of interest was the trial-defined primary bleeding event. Despite some differences in the definition of bleeding in the above studies, the primary bleeding event was a combination of major bleeding and clinically relevant nonmajor bleeding. The trial-defined primary ischemic event was a secondary outcome in our meta-analysis. For each trial, we recorded a risk estimate for the primary bleeding event and the primary ischemic event in the DOAC-based versus the VKAbased subgroups, both in the low bleeding risk (HAS-BLED $\leq 2$ ) and in the high bleeding risk subgroups (HAS-BLED $\geq 3$ ). The risk estimates are reported as hazard ratio (AUGUSTUS) or dichotomous frequency data (PIONEER-AF PCI, REDUAL PCI, and ENTRUST-AF PCI). We treated hazard ratio as relative risk (RR). The random effects model was used to obtain the pooled RR. Risk estimates between subgroups (HAS-BLED $\leq 2$ and $\geq 3$ ) were compared with a test of interaction. All analyses were performed with Comprehensive Meta-Analysis Version 2 (Biostat, Englewood, New Jersey, United States).

A total of 10,701 and 9,288 patients have been metaanalyzed for the primary and the secondary outcome, respectively (as ENTRUST-AF PCI does not provide major ischemic outcomes according to HAS-BLED score). The clinical data of the patients included in the analysis as well as the absolute rates of the trial-defined primary bleeding events stratified according to HAS-BLED score are shown in - Table 1. In subjects with HAS-BLED $\leq 2$, the cumulative RR for the trialdefined primary bleeding event in the DOAC-based versus the VKA-based antithrombotic regimen was 0.57 (95\% confidence intervals [CIs] 0.47 to 0.69 ), corresponding to a risk reduction received

April 27, 2020

accepted after revision

November 8, 2020

published online

December 31, 2020 (c) 2020. Thieme. All rights reserved. Georg Thieme Verlag KG,

Rüdigerstraße 14,

70469 Stuttgart, Germany
DOI https://doi.org/ $10.1055 / \mathrm{s}-0040-1721777$. ISSN 0340-6245. 
Table 1 Basic clinical characteristics and primary bleeding outcome of the included populations

\begin{tabular}{|l|l|l|l|l|}
\hline & Pioneer-AF PCI & REDUAL-PCI & AUGUSTUS & ENTRUST-AF PCI \\
\hline Age (y) & 70.1 & 70.2 & 70.7 & 69.5 \\
\hline ACS (\%) & 49.9 & 49.7 & 37.3 & 52 \\
\hline PCI (\%) & 100 & 100 & 76.1 & 100 \\
\hline $\begin{array}{l}\text { Primary bleeding event } \\
\text { (VKA, HAS-BLED } \leq 2 \text { ) (\%) }\end{array}$ & 19.4 & 22.5 & $11.1 \%$ (odds ratio DOAC vs. VKA 0.59) & 17.6 \\
\hline $\begin{array}{l}\text { Primary bleeding event } \\
\text { (VKA, HAS-BLED } \geq 3 \text { ) (\%) }\end{array}$ & 25.9 & 28.1 & $13.3 \%$ (odds ratio DOAC vs. VKA 0.72) & 21.9 \\
\hline $\begin{array}{l}\text { Primary bleeding event } \\
\text { (DOAC, HAS-BLED } \leq 2 \text { ) (\%) }\end{array}$ & 13.0 & 13.3 & $11.1 \%$ (odds ratio DOAC vs. VKA 0.59) & 10.9 \\
\hline $\begin{array}{l}\text { Primary bleeding event } \\
\text { (DOAC, HAS-BLED } \geq 3 \text { ) (\%) }\end{array}$ & 16.7 & 19.9 & $13.3 \%$ (odds ratio DOAC vs. VKA 0.72) & 20.1 \\
\hline
\end{tabular}

Abbreviations: ACS, acute coronary syndrome; DOAC, direct oral anticoagulant; ISTH, International Society on Thrombosis and Haemostasis; PCI, percutaneous coronary intervention; TIMI, Thrombolysis In Myocardial Infarction; VKA, vitamin K antagonist.

Note: Primary bleeding event was TIMI major and TIMI minor and bleeding requiring medical attention (PIONEER-AF PCI) or ISTH major and clinically relevant nonmajor bleeding (AUGUSTUS, REDUAL PCI, ENTRUST AF).

of $43 \%$ with the DOAC-based therapy. In subjects with HASBLED $\geq 3$, the respective cumulative RR was 0.69 (95\% CIs 0.61 to 0.78 ), corresponding to a reduction of $31 \%$ for the primary bleeding event with the DOAC-based therapy ( - Fig. 1A). There was no significant difference of the RR estimates between the subgroups of HAS BLED $\leq 2$ and HAS-BLED $\geq 3$ ( $Q$ value 3.03 , $p=0.082$ ). No significant heterogeneity across the four trials regarding the primary bleeding event was observed $\left(I^{2} 22.75 \%\right.$, $p=0.248$ ). Sensitivity analysis after excluding ENTRUST AF PCI trial confirmed the findings, with a cumulative RRs of 0.57
(95\% CIs 0.46 to 0.69 ) in the HAS BLED $\leq 2$ subgroup and 0.66 ( $95 \%$ CIs 0.58 to 0.75 ) in the HAS-BLED $\geq 3$ subgroup. There was no significant change of the risk for the trial-defined primary ischemic event in the DOAC-based versus the VKAbased regimen ( - Fig. 1B); similarly, no difference of the RR estimates between the two subgroups of HAS BLED was observed ( $Q$ value $0.89, p=0.35$ ). We observed significant heterogeneity across the three trials regarding the primary ischemic event $\left(I^{2} 59.17 \%, p=0.032\right)$. Bias analysis with funnel plots and the fail-safe $\mathrm{N}$ test showed absence of significant
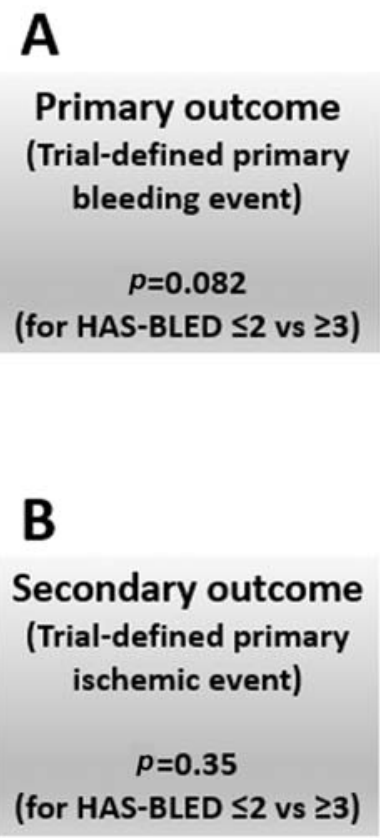

$\begin{array}{lcc}\text { Study } & \text { HAS-BLED } & \text { RR } \\ \text { PIONEER } & \leq \mathbf{2} & 0.62 \\ \text { REDUAL } & \leq \mathbf{2} & 0.53 \\ \text { AUGUSTUS } & \leq \mathbf{2} & 0.59 \\ \text { ENTRUST } & \mathbf{\leq 2} & 0.57 \\ \quad \text { Subtotal } & & \mathbf{0 . 5 7} \\ \text { PIONEER } & \geq \mathbf{3} & 0.57 \\ \text { REDUAL } & \geq \mathbf{3} & 0.64 \\ \text { AUGUSTUS } & \geq \mathbf{3} & 0.72 \\ \text { ENTRUST } & \geq \mathbf{3} & 0.92 \\ \quad \text { Subtotal } & & \mathbf{0 . 6 9} \\ \text { Total } & & \mathbf{0 . 6 4}\end{array}$

$\begin{array}{lcc}\text { Study } & \text { HAS-BLED } & \text { RR } \\ \text { PIONEER } & \mathbf{\leq 2} & 1.20 \\ \text { REDUAL } & \mathbf{\leq 2} & 0.73 \\ \text { AUGUSTUS } & \mathbf{\leq 2} & 0.85 \\ \quad \text { Subtotal } & & \mathbf{0 . 8 3} \\ \text { PIONEER } & \geq \mathbf{3} & 1.13 \\ \text { REDUAL } & \geq \mathbf{3} & 1.27 \\ \text { AUGUSTUS } & \geq \mathbf{3} & 0.82 \\ \quad \text { Subtotal } & & \mathbf{1 . 0 2} \\ \text { Total } & & \mathbf{0 . 9 3}\end{array}$

$95 \% \mathrm{Cls}$

$0.36-1.07$

$0.39-0.72$

$0.43-0.81$

$0.33-0.98$

0.47-0.69

$0.42-0.78$

$0.52-0.77$

$0.59-0.88$

$0.68-1.26$

0.61-0.78

$0.52-0.77$

Z-value
-1.72
-4.12
-3.33
-2.03
-5.90
-3.53
-4.54
-3.22
-0.50
-6.14
-4.64

$p$-value
0.086
$<0.001$
0.001
0.042
$<0.001$
$<0.001$
$<0.001$
0.001
0.618
$<0.001$
$<0.001$

Relative risk and $95 \% \mathrm{Cls}$
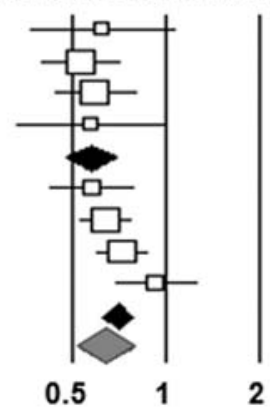

Favours DOAC Favours VKA

$95 \% \mathrm{Cls}$

0.48-3.03

0.52-1.03

0.69-1.05

0.60-1.15

$0.66-1.92$

1.00-1.61

0.71-0.95

0.77-1.35

$0.76-1.15$

Z-value
0.39
-1.81
-1.52
-1.13
0.44
1.94
-2.67
$\mathbf{0 . 1 4}$
-0.63
p-value
0.694
0.071
0.129
0.260
0.661
0.052
0.008
0.885
0.526

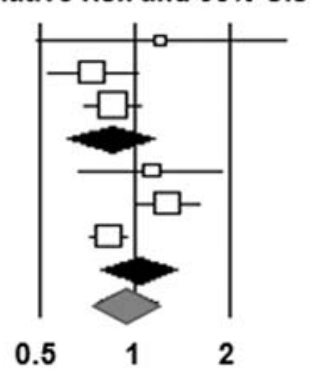

Favours DOAC Favours VKA

Fig. 1 Cumulative relative risk and 95\% confidence interval for the primary bleeding event (A) and the primary ischemic event (B) in direct oral anticoagulant (DOAC)-based dual antithrombotic therapy compared with vitamin K antagonist (VKA)-based triple antithrombotic therapy, stratified according to the bleeding risk (HAS-BLED score). 
publication bias as far as the primary bleeding event is concerned.

Decision regarding the optimal antithrombotic regimen in AF patients taking anticoagulants who need additional antiplatelet therapy after PCI comprises a challenge and is mainly driven by estimating the balance between bleeding and ischemic risk. ${ }^{3}$ Our meta-analysis shows that a DOAC-based DAT is associated with a lower risk of bleeding not only in subjects with a high bleeding risk (HAS-BLED $\geq 3$ ), but also in patients with a lower bleeding risk (HAS-BLED $\leq 2$ ). Although a few years ago guidelines suggested triple therapy up to 6 months post-PCI in low-bleeding risk acute coronary syndrome patients with $\mathrm{AF}^{10}$ our analysis clearly shows that DAT is beneficial without compromising efficacy even in this subgroup. This is reflected in the recent 2020 European Society of Cardiology Guidelines for the diagnosis and management of AF, which recommend that triple therapy should not be taken for more than 1 month after PCI in acute coronary syndrome patients with $\mathrm{AF}$, independent of the bleeding risk. ${ }^{11}$

Apart from inherent limitations of the type of analysis performed, it is notable that data obtained from the AUGUSTUS trial refer to a DOAC-based versus a VKA-based strategy with both strategies including DAT and triple therapy subgroups. Moreover, $23.9 \%$ of the AUGUSTUS patients included in our analysis underwent no $\mathrm{PCl}$ at baseline. ${ }^{7}$ Meta-analysis for individual components of the primary bleeding or ischemic events (i.e., intracranial hemorrhage or stent thrombosis, etc.) has not been performed as none of the trials provide such data stratified according to HAS-BLED score.

With the foregoing limitations, we conclude that in AF patients undergoing PCI, a DAT with DOAC plus P2Y12 inhibitor is safer compared with a VKA-based triple antithrombotic therapy irrespectively of the baseline bleeding risk. It should be kept in mind that bleeding risk is dynamic and may change over time, and that the change of HAS-BLED score during follow-up may be more predictive for major bleeding than baseline HAS-BLED score. ${ }^{12} \mathrm{~A}$ high bleeding risk (HAS-BLED $\geq 3$ ) should activate clinicians to address modifiable bleeding risk factors and review the patients earlier and more frequently. ${ }^{11}$

\section{Conflict of Interest}

None declared.

\section{References}

1 Lip GY, Frison L, Halperin JL, Lane DA. Comparative validation of a novel risk score for predicting bleeding risk in anticoagulated patients with atrial fibrillation: the HAS-BLED (Hypertension,
Abnormal Renal/Liver Function, Stroke, Bleeding History or Predisposition, Labile INR, Elderly, Drugs/Alcohol Concomitantly) score. J Am Coll Cardiol 2011;57(02):173-180

2 Borre ED, Goode A, Raitz G, et al. Predicting thromboembolic and bleeding event risk in patients with non-valvular atrial fibrillation: a systematic review. Thromb Haemost 2018;118(12): 2171-2187

3 Benetou DR, Varlamos C, Mpahara A, Alexopoulos D. Double or triple antithrombotic treatment in atrial fibrillation patients with acute myocardial infarction undergoing percutaneous coronary intervention. Am J Cardiovasc Drugs 2021;21(01): $11-20$

4 Dewilde WJ, Oirbans T, Verheugt FW, et al;WOEST study investigators. Use of clopidogrel with or without aspirin in patients taking oral anticoagulant therapy and undergoing percutaneous coronary intervention: an open-label, randomised, controlled trial. Lancet 2013;381(9872):1107-1115

5 Gibson CM, Mehran R, Bode C, et al. Prevention of bleeding in patients with atrial fibrillation undergoing PCI. N Engl J Med 2016;375(25):2423-2434

6 Cannon CP, Bhatt DL, Oldgren J, et al;RE-DUAL PCI Steering Committee and Investigators. Dual antithrombotic therapy with dabigatran after PCI in atrial fibrillation. N Engl J Med 2017;377 (16):1513-1524

7 Lopes RD, Heizer G, Aronson R, et al;AUGUSTUS Investigators. Antithrombotic therapy after acute coronary syndrome or $\mathrm{PCI}$ in atrial fibrillation. N Engl J Med 2019;380(16):1509-1524

8 Vranckx P, Valgimigli M, Eckardt L, et al. Edoxaban-based versus vitamin $\mathrm{K}$ antagonist-based antithrombotic regimen after successful coronary stenting in patients with atrial fibrillation (ENTRUST-AF PCI): a randomised, open-label, phase 3b trial. Lancet 2019;394(10206):1335-1343

9 Gargiulo G, Goette A, Tijssen J, et al. Safety and efficacy outcomes of double vs. triple antithrombotic therapy in patients with atrial fibrillation following percutaneous coronary intervention: a systematic review and meta-analysis of non-vitamin $\mathrm{K}$ antagonist oral anticoagulant-based randomized clinical trials. Eur Heart J 2019;40(46):3757-3767

10 Valgimigli M, Bueno H, Byrne RA, et al;ESC Scientific Document Group ESC Committee for Practice Guidelines (CPG) ESC National Cardiac Societies. 2017 ESC focused update on dual antiplatelet therapy in coronary artery disease developed in collaboration with EACTS: the Task Force for dual antiplatelet therapy in coronary artery disease of the European Society of Cardiology (ESC) and of the European Association for Cardio-Thoracic Surgery (EACTS). Eur Heart J 2018;39(03):213-260

11 Hindricks G, Potpara T, Dagres N, et al;ESC Scientific Document Group. 2020 ESC Guidelines for the diagnosis and management of atrial fibrillation developed in collaboration with the European Association of Cardio-Thoracic Surgery (EACTS). Eur Heart J 2020; $\mathrm{x}: 612$

12 Chao TF, Lip GYH, Lin YJ, et al. Incident risk factors and major bleeding in patients with atrial fibrillation treated with oral anticoagulants: a comparison of baseline, follow-up and delta HAS-BLED scores with an approach focused on modifiable bleeding risk factors. Thromb Haemost 2018;118(04):768-777 